\title{
Where does damage lead to enhanced food aversion: the ventral pallidum/substantia innominata or lateral hypothalamus?
}

\author{
Howard C. Cromwell and Kent C. Berridge \\ Department of Psychology, The University of Michigan, Ann Arbor, MI 48104-1687 (USA)
}

(Accepted 20 April 1993)

Key words: Ventral pallidum/substantia innominata; Lateral hypothalamus; Globus pallidus; Quinolinic acid; Ibotenic acid; Excitotoxin lesion; Aphagia; Feeding behavior; taste aversion

\begin{abstract}
It is well known that lesions of the lateral hypothalamus (LH) produce aphagia. Several previous studies have reported that lateral hypothalamus damage produces food aversion in addition to aphagia. However, damage to other regions near the LH also produce aphagia and enhanced aversion. The purpose of this study was to resolve where the site or sites for aversion-inducing lesions is/are located. Small, bilateral excitotoxin lesions (QUIN, $10 \mu \mathrm{g}$ in $1 \mu \mathrm{l}$ or IBO, $15 \mu \mathrm{g}$ in $1 \mu \mathrm{l}$ ) or bilateral sham injections of vehicle were made into the globus pallidus (GP), the ventral pallidum/substantia innominata (VP/SI) or the lateral hypothalamus (LH). Affective reactions to taste were elicited by infusing sucrose solutions $(1 \mathrm{M})$ into the mouth via chronic oral cannulae. The number of aversive responses (gapes, chin-rubbing, head-shaking and forelimb flails) emitted was tallied. Individual lesions were mapped and a single 'necessary and sufficient' site for damage-induced aversion was identified (the area of overlapping damage common to all rats that showed enhanced aversive reactions). To identify the lesions, two lesion-mapping techniques were used: (1) a conventional neuron-counting procedure in which an attempt is made to count all neurons within a brain region, and (2) a new modified 'fractionator' procedure consisting of exhaustive $400 \times$ magnification counts at point locations within a brain region. Results indicated that aversive reactions to food are enhanced only following bilateral neuron loss $(>70 \%)$ from the caudal ventromedial VP/SI alone. This shared site has a lateral diameter of $1.0 \mathrm{~mm}$, a dorsoventral diameter of $0.5 \mathrm{~mm}$ and a rostrocaudal diameter of $1.0 \mathrm{~mm}$. Damage restricted to the LH never produced enhanced aversion even when it produced aphagia. The crucial region for aversion is located ventral and medial to the globus pallidus and dorsal and lateral to the lateral hypothalamus.
\end{abstract}

\section{INTRODUCTION}

Large electrolytic lesions of the lateral hypothalamus (LH) have long been known to lead to aphagia and adipsia $^{2,9,38}$. Aphagia also results after excitotoxin lesions of the LH that spare fibers of passage ${ }^{26,37,43}$. In addition, especially after rostral and far-lateral electrolytic LH lesions, rats may show active aversion to normally palatable food, by engaging in food spillage, gaping, and forelimb flailing ${ }^{35,36,39}$. Not all aphagic rats show enhanced aversive reactions to food but some do. For example, in one study rats with bilateral LH damage were presented with a sugar solution and, 'displayed vigorous withdrawal behaviors... when the stimulus was brought to the mouth; the rat typically oriented away from the stimulus, blocked its mouth with its forepaws and tightly closed its lips ${ }^{36}$. In another study, when a palatable cookie mash was spooned into the mouth, rats with $\mathrm{LH}$ damage were reported to, 'display reactions of aversion and actually avoid contact with these stimuli on repeated presentations. ${ }^{35}$.

The lateral hypothalamus is not the sole basal forebrain region in which lesions produce aphagia and enhanced aversion. For example, electrolytic or excitotoxin lesions of the globus pallidus (GP) have been suggested to produce aphagia as severe as that produced by $\mathrm{LH}$ damage ${ }^{16,26,27,34}$. Excitotoxin lesions of the basal forebrain within either the ventral pallidum/ substantia innominata (VP/SI) or nucleus basalis also have been suggested to produce aphagia ${ }^{5,72}$. Finally, many of these extra-hypothalamic lesions also produce enhanced aversive reactions (gapes, chin rubs, etc.) to normally palatable tastes such as sucrose $e^{7,13,42}$.

The ability of both LH-centered lesions and lesions centered upon other basal forebrain sites to enhance food aversion as well as to produce aphagia raises the 
question of whether aversion-producing lesions act upon the same or separate neural systems. Are there multiple sites in which neuronal cell loss creates enhanced aversion for food? Or do these ostensibly different lesions actually depend upon damage to a single anatomical population of neurons?

The possibility that both types of lesions owe their food aversion effects to a shared site of damage is made plausible by the fact that most of the previous studies have used large and overlapping lesions. For example, the dorsal tip of the electrolytic LH lesions made by Teitelbaum and Epstein ${ }^{39}$ ascended into the internal capsule, passing through the posterior ventral pallidal region. Schallert and Whishaw ${ }^{35}$ identified two qualitatively different types of $\mathrm{LH}$ aphagia. Only one of these, active aphagia, was accompanied by aversive reactions to food. Active aphagia was produced only by anterior LH lesions in which damage invaded the ventral pallidum/substantia innominata region, and it is possible that some behavioral ' $\mathrm{LH}$ ' effects were due to pallidal damage. Conversely, previous studies of aversion produced by non-LH basal forebrain lesions have often produced incidental damage in the lateral hypothalamus, so it is possible that some 'ventral pallidal or nucleus basalis' effects are due to $\mathrm{LH}$ damage da, $^{5,42}$.

The purpose of the present study was to ascertain whether LH, GP and VP/SI lesions that induce aversion, in addition to aphagia, do so by damaging separate sites or one common site. In order to do this, we compared the aversion-enhancing effects of small excitotoxin lesions that were centered in either the $\mathrm{LH}$ or the VP/SI. The boundaries of lesions that produced enhanced aversion in a taste reactivity paradigm ${ }^{17}$ were carefully mapped using an objective and sensitive technique: a modification of the fractionator technique of Gundersen et al. ${ }^{20}$. Areas of damage that were shared by aversion-enhancing lesions were identified by a 'subtraction analysis'. Our results suggest that there is only one region in the basal forebrain in which neuron loss enhances aversion. This site lies outside the lateral hypothalamus itself, but within the region damaged by many 'LH' lesions: a site at the ventromedial edge of the ventral pallidum/substantia innominata (vmVP/ SI).

\section{MATERIAL AND METHODS}

\section{Subjects}

Subjects were 52 male Sprague-Dawley rats (Charles-River) weighing $300 \pm 50 \mathrm{~g}$ at the beginning of the experiment. They were housed singly under a $12: 12 \mathrm{~h}$ light: dark cycle. Water and food were freely available.

\section{Surgical procedures}

Rats were anesthetized using first halothane (within a desiccator) and then methoxy-fluorane given through an air anesthesia system for small animals ${ }^{25}$. Excitotoxin injections intended to destroy neurons within the globus pallidus were centered at the coordinates: AP $1.0 \mathrm{~mm}$ posterior to bregma; $\mathrm{L} \pm 2.8 \mathrm{~mm}$ lateral to the midline; $\mathrm{V} 7.5$ $\mathrm{mm}$ ventral to the skull (quinolinic acid, $10 \mu \mathrm{g}, n=10$ rats). Excitotoxin injections intended to destroy neurons within the ventral pallidum/substantia innominata region were centered at the coordinates: AP $1.0 \mathrm{~mm}$ posterior to bregma; $\mathrm{L} \pm 2.8 \mathrm{~mm}$ lateral to the midline; V $8.0 \mathrm{~mm}$ ventral to the skull (quinolinic acid, $10 \mu \mathrm{g}, n=11$ rats; ibotenic acid, $15 \mu \mathrm{g}, 3$ rats). Injections intended to destroy neurons in the lateral hypothalamus were centered at the coordinates: AP $2.7 \mathrm{~mm}$ posterior from bregma; $\mathrm{L} \pm 1.2 \mathrm{~mm}$ lateral to the midline; and V $8.5 \mathrm{~mm}$ ventral to the skull (ibotenic acid, $15 \mu \mathrm{g}$, $n=9$ rats; quinolinic acid $10 \mu \mathrm{g}, 3$ rats). The actual center of individual lesions varied from rat to rat (standard deviation (S.D.) =

(A)

HEDONIC REACTIONS
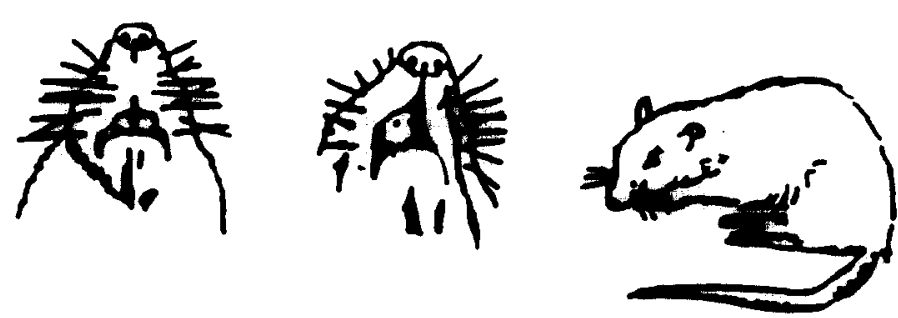

(B)

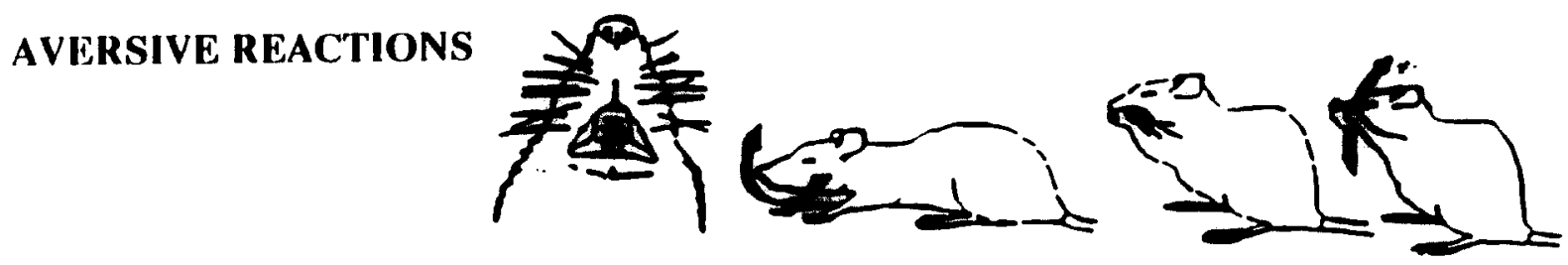

Fig. 1. Affective reactions to taste. Hedonic reactions (top) are elicited by sucrose and other palatable tastes. Hedonic reactions include rhythmic midline tongue protrusion, nonrhythmic lateral tongue protrusion, and paw lick. Aversive reactions (bottom) are elicited by quinine and other non-palatable tastes. Aversive reactions include gape, head-shake, face wash, and forelimb flail. 
$0.8 \mathrm{~mm}$ ). All injections were $1 \mu 1$ and the vehicle solution was always phosphate-buffered saline $(\mathrm{pH}=7.4)$. Ibotenic acid was used as the primary neurotoxin for the $\mathrm{LH}$ lesions because previous work has shown that this neurotoxin reliably produces aphagia and adipsia after being injected into the $\mathrm{LH}^{43}$. Sham-operated controls received infusions of PBS alone (pallidal/SI, $n=8 ; \mathrm{LH}, n=8$ ). Each injection was made over a 3-min period, and the needle was left in place for an additional $5 \mathrm{~min}$. Diazepam $(8 \mathrm{mg} / \mathrm{kg}$ ) was given within $30 \mathrm{~min}$ of the injection. Atropine sulfate $(3 \mathrm{mg} / \mathrm{kg}$, i.p.) and bicillin $(30,000$ units, i.m.) were given prior to surgery.

Each rat was implanted with bilateral chronic oral cannulae to allow for the infusion of taste solutions into the mouth. These cannulae (heat-flared PE-50 tubing) enter the mouth lateral to the first maxillary molar and exit the head near the dorsolateral boundary of the skull, where they are anchored with skull screws and acrylic cement.

Thirty minutes post-surgery, a second injection of diazepam ( 8 $\mathrm{mg} / \mathrm{kg}$ ) was given to reduce any convulsive activity (although obvious convulsions were not usually observed). All rats were given $250 \mathrm{ml}$ of cereal mash (commercial baby cereal mixed with water), 5 chow pellets and $40 \mathrm{ml}$ of water per day (feeding between 17.00 and 19.00 h). Intake was monitored by counting the number of pellets consumed, the approximate amount of mash eaten, and the amount of water drank within a $24-\mathrm{h}$ period. Rats were weighed daily. If a rat lost weight compared to his pre-surgical weight, then an intubation procedure was initiated. For each $5 \mathrm{~g}$ of weight lost, the rat was intubated with $12 \mathrm{ml}$ of vitamin-supplemented sweetened milk solution, up to 3 intubations per day. Rats were considered aphagic if neither chow pellets nor cereal mash was eaten.

\section{Behavioral testing}

Using the taste reactivity paradigm devised by Grill and Norgren ${ }^{17}$, taste reactions were videotaped to oral infusions of sucrose $(1 \mathrm{M})$ or quinine (HCL $3 \times 10^{-4} \mathrm{M}$ ), given in random order, beginning $48 \mathrm{~h}$ following surgery. The initial trial used distilled water infusions in order to habituate rats to the procedure. A delivery tube was connected to an oral cannula of the rat, and following a 5-min habituation period, $1 \mathrm{ml}$ of the test solution was infused directly into the mouth over a 1-min period. If multiple stimuli were given in one day, there was a 2 -h interval between the tests. Tests were run between 16.00 and 20.00 h (i.e. shortly prior to lights out). On each trial, a rat was placed in a transparent test chamber, the floor of which was suspended over an angled mirror. The mirror reflected a view of the rat's mouth and forepaws to the videocamera.

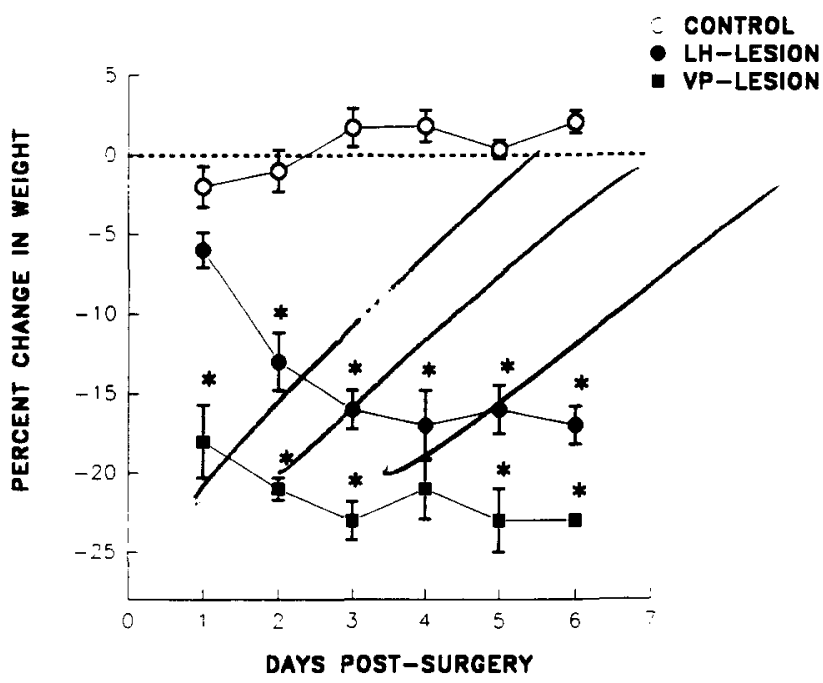

Fig. 2. Cumulative percent weight change from pre-surgery weight. There was no significant difference between the LH-controls or VP/SI-controls so these groups were combined into one control group. Body weights of the GP lesion group did not differ from controls and is not shown. ${ }^{*}$ denotes significance at $P<0.01$.
Sucrose elicits primarily hedonic responses from normal rats and quinine elicits primarily aversive responses. $24 \mathrm{~h}$ after the distilled water taste trial, each rat was given sucrose $(1 M)$ or quinine $\left(3 \times 10^{-4} \mathrm{M}\right)(1 \mathrm{ml}$ delivered for a 1 -min period) on separate days in counterbalanced order. For aphagic rats receiving intubations, taste trials were conducted at least $2 \mathrm{~h}$ after the last intubation.

\section{Taste reactivity scoring}

Videotapes were scored in slow motion (frame by frame to $1 / 10$ the normal speed) for the occurrence of aversive and hedonic reactions (see Fig. 1.) Aversive actions include: gapes, large openings of the mouth and jaw lasting about $125 \mathrm{~ms}$; chin-rubbing, bringing the chin in direct contact with the floor and projecting the body forward; face-washing, either a single wipe with the paws over the face or a bout of several wipes; forelimb flails, shaking of the forelimb back and forth; and rapid head shaking.

The following hedonic reactions were scored: lateral tongue protrusions (non-rhythmic) lasting about $160 \mathrm{~ms}$; rhythmic tongue protrusions: these protrude along the midline with a cycle length of roughly $160 \mathrm{~ms}$; and paw-licking. Each action was scored and counted using criteria described in Grill and Berridge ${ }^{18}$.

\section{Histology}

At the conclusion of the behavioral testing, each rat was deeply anesthetized and intracardially perfused. Perfusions were completed with $0.9 \%$ saline followed by $10 \%$ formalin in PBS, $\mathrm{pH}=7.4$. Brains were removed and stored in a $30 \%$ sucrose solution (10\% formalin). Rats that died prior to perfusion were decapitated, and the brain was removed and soaked in formalin for at least 7 days. The brains were blocked, frozen, and sliced in $30 \mu \mathrm{m}$ slices using a sliding microtome. Slices were mounted directly onto gelatin-coated slides for Niss staining. The slides were dipped in xylene and ethanol baths $(70 \%$, 95\% and 100\%) for cleaning and defatting. After being dipped in Cresyl violet, the slides were taken through the final alcohols and xylenes prior to coverslipping using permount.

\section{Analysis 1: conventional neuron count for lesion mapping}

Three anterior-posterior (AP) levels per brain were counted. Slices for each rat were chosen to approximate each level. The anterior level was located at roughly $-0.3 \mathrm{~mm}$ posterior from bregma. The landmarks associated with the anterior level include crossing of the anterior commissure as well as a rounded fornix. The medial level was located $-1.3 \mathrm{~mm}$ from bregma. Landmarks associated with this area include a triangular septal area with an enlarged globus pallidus. The posterior level was roughly located at $-2.8 \mathrm{~mm}$ from bregma, and was characterized by the flattened hippocampal commissure, the appearance of the CA3 field of Ammon's horn, the disappearance of the ventral pallidum, and the appearance of the lateral hypothalamus medially and the anterior amygdala laterally. Counts of neurons were made using a light microscope (Leitz Laborlux S). To determine the neuronal density within striatopallidal and lateral hypothalamic zones, neuron cell counts were completed using a eyepiece reticule $(10 \mathrm{~mm}$ by $10 \mathrm{~mm}$ divided into $1.0 \mathrm{~mm}$ squares) placed into a $10 \times$ eyepiece. The light microscope analysis was completed using a $20 \times$ objective (total magnification $200 \times$ ). A neuron was defined for scoring purposes as an intact circular- or ovoid-shaped object with a light purple interior stain, at least $10 \mu \mathrm{m}$ in diameter (i.e. the diameter of one-fifth of an individual $50-\mu \mathrm{m}$ grid). The nucleus was not required in order to be counted as a neuron. At each of these $3 \mathrm{AP}$ levels, neuron counts were made bilaterally in five different regions: the centromedial striatum; the centrolateral striatum; the ventrolateral region; the globus pallidus; and the basal forebrain region which includes the VP/SI at the anterior level and the lateral hypothalamus at the posterior level.

\section{Statistics}

Group taste reactions were compared using the Mann-Whitney $U$ non-parametric test. Means of regional neuron counts between each lesion group and each sham injected control group were compared using both the single factor randomized ANOVA and the Newman-Keuls test. Means of regional neuron counts between lesion groups were compared using the Newman-Keuls test. 


\section{RESULTS}

Food intake and body weight

$G P$

None of the rats that received excitotoxin injections into the globus pallidus showed any significant loss of feeding or drinking behavior. During the first $24-72 \mathrm{~h}$ post-surgery, the main form of food eaten was cereal mash in these animals. Feeding upon chow pellets usually began within 3 days. Body weights did not vary significantly from controls.

\section{$L H$}

Eight out of 12 rats that received ibotenic acid $(n=6)$ or quinolinic acid $(n=2)$ injections into the $\mathrm{LH}$ were aphagic and adipsic. The average length of aphagia and adipsia was 4.9 days (range 2-8 days) and the average weight loss was $57 \mathrm{~g}$ (a cumulative average decrease of $14 \%$ compared to controls, $F_{1,14}=16.91$, $P<0.01$, see Fig. 2).

\section{$V P / S I$}

Eight out of 14 rats that received quinolinic acid injections into the VP/SI were aphagic and adipsic. The average length of aphagia and adipsia was 5 days (range, 2-7 days). The average weight loss was $64 \mathrm{~g}$ over a 6-day period (average cumulative percent decrease $=21 \%$ compared to 5 controls, $F_{1,11}=31.23$, $P<0.001$, see Fig. 2). The aphagia following VP/SI excitotoxin injection was most intense during the first $24 \mathrm{~h}$ post-surgery when rats lost on average $>40 \mathrm{~g}$ in this time period (see Fig. 2). Other changes seen in the home cage included motor impairments such as akinesia and postural deficits. Some rats showed chronic limb extension, mainly of the hindlimbs extending out 90 degrees from the main axis of the body, and sensory orientation deficits.

\section{Aversive and hedonic taste reactivity}

In order to maximize the detection of aversion enhancement, only taste reactivity data from the period of actual aphagia were analyzed from rats with lesions. The LH lesion group $(n=8)$ did not show enhanced aversive reactions, even though these rats were aphagic. Instead these rats showed a trend towards suppression of both aversive responses to quinine $\left(3 \times 10^{-4} \mathrm{M}\right)$ (11.2 aversive responses to 17.2 aversive responses in controls) and hedonic responses to palatable sucrose (1 M) (5.5 ingestive responses compared to 12 ingestive responses in control animals). This trend did not reach significance in this analysis of reactions to sucrose and

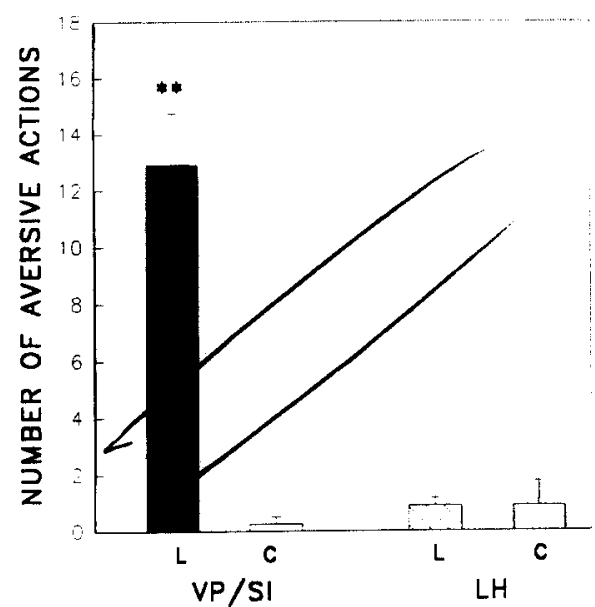

Fig. 3. Number of aversive taste reactions per 1-min taste trial of $1 \mathrm{M}$ sucrose solution. $L$ denotes rats which received excitotoxin injection into a particular subregion and $C$ denotes sham-injected control animal. ${ }^{*}$ denotes significance at $P<0.001$.

quinine solutions, although the suppression of hedonic and aversive reactions was significant in a more exhaustive analysis that included reactions to other tastes $^{8}$. LH rats emitted no aversive reactions to palatable sucrose. By contrast, rats that received excitotoxin injections into the VP/SI region had a significant increase in the number of aversive responses to sucrose (1 M) compared to sham-injected control rats $(n=8$, $13.5 \pm 2$ to 0.00 responses, Mann-Whitney $U$ test, $P<0.001$, see Fig. 3) and compared to LH rats (13.5 to 0.00 responses, Mann-Whitney $U, P<0.01$ ).

\section{Lesion identification}

Rats that received excitotoxin injection into the GP had neuronal depletion $\geq 60 \%$ bilaterally from the ventromedial GP. Aphagic rats that received excitotoxic acid injections into the $\mathrm{LH}$ had $>70 \%$ neuron loss bilaterally from the LH (see Table I). Aphagic rats that received injections into the VP/SI had significant neuron depletion in the ventral pallidum/substantia innominata (VP/SI, mean $=66 \%$ ), the globus pallidus $(\mathrm{GP}$, mean $=47 \%)$ and the lateral hypothalamus ( $\mathrm{LH}$, mean $=53 \%)($ see Table I). However, a rat-by-rat analysis showed that only one zone had greater than $50 \%$ depletion in all rats that showed enhanced aversion: the VP/SI region. The minimum level of damage found in rats that showed enhanced aversion by this conventional neuron count analysis was 55\% average neuron depletion. This level of depletion appeared to be the approximate minimum threshold of damage for enhanced aversion. Only 5 out of 8 of the 'aversive' rats had $>50 \%$ depletion in the GP and only 4 out of 8 rats had $>50 \%$ depletion in the LH. Even though 
TABLE I

Regions with a significant neuron depletion in rats receiving excitotoxin injections into the GP, LH or VP / SI

The excitotoxin is given following the injection site (ibotenic or quinolinic). Only the VP/SI region (in italics) was damaged in every rat that showed aversion. ${ }^{*}$ and ${ }^{* *}$ are given to denote significance at $P<0.05$ and $P<0.01$ levels, respectively. Statistical analysis was completed using region count averages for each group with the Newman-Keuls test.

\begin{tabular}{llrl}
\hline Injection site (n) & Region & \multicolumn{1}{c}{ Neuron } & \% Reduced \\
\hline GP-QUIN (8) & GP-LEFT & $22 \pm 3$ & $72 *$ \\
GP-SHAM (8) & GP-LEFT & $79 \pm 17$ & \\
GP-QUIN (8) & GP-RIGHT & $32 \pm 6$ & $63 *$ \\
GP-SHAM & GP-RIGHT & $86 \pm 25$ & \\
VP/SI-QUIN (8) & GP-LEFT & $30 \pm 11$ & $62 *$ \\
VP/SI-SHAM (7) & GP-LEFT & $79 \pm 17$ & \\
VP/SI-QUIN (8) & GP-RIGHT & $60 \pm 21$ & 30 \\
VP/SI-SHAM (7) & GP-RIGHT & $86 \pm 25$ & \\
VP/SI-QUIN (8) & VP-LEFT & $52 \pm 17$ & $60 * *$ \\
VP/SI-SHAM (7) & VP-LEFT & $131 \pm 22$ & \\
VP /SI-QUIN (8) & VP-RIGHT & $27 \pm 6$ & $72 * *$ \\
VP/SI-SHAM (7) & VP-RIGHT & $130 \pm 25$ & \\
VP/SI-QUIN (8) & LH-LEFT & $72 \pm 14$ & $47 *$ \\
VP/SI-SHAM (7) & LH-LEFT & $137 \pm 15$ & \\
VP/SI-QUIN (8) & LH-RIGHT & $62 \pm 10$ & $58 * *$ \\
VP/SI-SHAM (7) & LH-RIGHT & $146 \pm 21$ & \\
LH-IBOTENIC (8) & LH-LEFT & $30 \pm 21$ & $81 * *$ \\
LH-SHAM (8) & LH-LEFT & $154 \pm 17$ & \\
LH-IBOTENIC (8) & LH-RIGHT & $37 \pm 17$ & $73 * *$ \\
LH-SHAM (8) & LH-RIGHT & $146 \pm 21$ & \\
\hline
\end{tabular}

the highest average levels of neuronal depletion occurred in the LH $(81 \%)$ rather than the VP/SI, no aversion was produced by $\mathrm{LH}-\mathrm{centered}$ lesions.

Fibers of passage. Destruction of ascending dopamine fibers of passage through the $\mathrm{LH}$ is well known to produce aphagia ${ }^{40}$. Therefore it is imperative to rule out the possibility that damage to fibers of passage contributed to behavioral changes. In order to determine whether fibers of passage were destroyed by excitotoxin lesions, leading to neuron loss elsewhere, we examined target structures of fibers for evidence of gliosis and neuron loss. Outside of the GP, VP/SI, or LH damage was rare. Less than $20 \%$ of the rats showing enhanced aversion had significant neuron depletion or gliosis in the striatum, thalamus, hippocampus, or medial cortex. Finally, it is especially unlikely that damage to mesotelencephalic dopamine fibers of passage contributed to the enhancement of aversion, since it has been shown that the aphagia which is produced by bilateral 6-OHDA lesions does not enhance aversive reactions to taste ${ }^{6}$.

The observation that damage within the VP/SI region is the only histological feature to unite all rats that show enhanced aversion indicates that damage within this region may be the chief cause of all enhanced aversion. However, it does not tell us the location within the VP/SI region of the crucial site of damage which produces aversion.

\section{Analysis 2: modified fractionator technique}

In order to identify the crucial site within the ventral pallidum where cell loss enhances aversive reactions to food, a second analysis was made based on the fractionator method of Gundersen et al. ${ }^{20}$, which was modified to allow application to lesion mapping. The modified fractionator method is a technique that allows precise and objective identification of a lesion's location, and the size and shape of its boundaries.

Our application of the fractionator method was done in three steps. First, a control baseline analysis of normal neuronal density was made using the fractionator method for 8 fractions within the ventral pallidum and the LH areas of interest ( $n=8$ rats). Second, the lesion of each rat that showed enhanced aversion was mapped using the fractionator method in order to identify (a) the location of center of the lesion, (b) the degree of neuronal depletion at the center of the lesion and (c) the location of the boundaries of the lesion 'core' (defined as the area of $>80 \%$ neuron depletion) and the lesion 'shell' (defined as the area of $>50 \%$ neuron depletion). An analysis of lesion 'cores' and 'shells' indicated that the approximate distance between the perimeter of $>80 \%$ neuron depletion and 50\% depletion was $250-500 \mu \mathrm{m}$. Thus, the apparent 'threshold' level of aversion-inducing damage (55\%) was always contained within this radius between the lesion core ( $80 \%$ neuron depletion) and shell (50\% neuron depletion) borders. Third, the location and boundaries of the crucial aversion site (defined as the site which must be damaged in order to produce aversion) were mapped by subtracting unshared regions of damage from the area of overlap among lesions that induced aversion. Our rationale for identifying the crucial site was chosen in order to define the site in which damage was a necessary and sufficient cause for enhanced aversion. Only a site which was damaged in every rat that showed enhanced aversion could be a necessary site for aversive enhancement; sites that were spared in any aversive rat could not be regarded as necessary for the enhancement of aversion. Only a site in which damage above 55\% was always accompanied by aversion could be considered sufficient in producing enhanced aversion: sites that had bilateral damage $>55 \%$ in any rat that showed normal taste reactions could not be regarded as sufficient for the enhancement of aversion. 


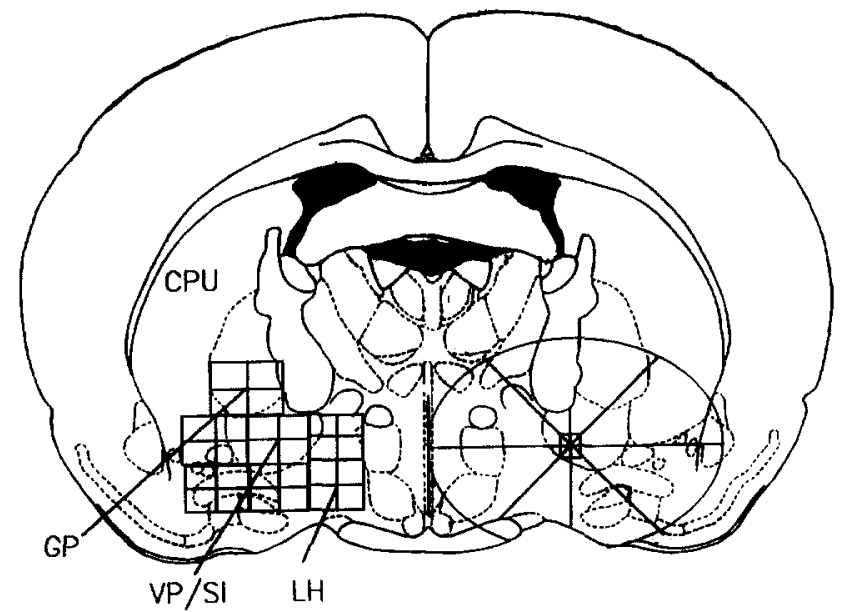

Fig. 4. Left side: location of fractions in which neuron count samples were taken for the modified fractionator technique. Right side: example of lesion boundary analysis along 8 radial arms from the center of a VP lesion. Neuron counts were continued along each arm until either the outer boundary of the lesion edge or the circumference was reached ( $2.5 \mathrm{~mm}$ from center), whichever came first.

\section{Stage 1. Determination of baseline neuron numbers}

For each of the 28 fractions (see Fig. 4), an exhaustive neuronal count was made of a $250 \times 250 \times 30 \mu \mathrm{m}$ 'core sample'. This was done by delineating a $250 \mu \mathrm{m}^{2}$ of a $30 \mu \mathrm{m}$ tissue section (randomly chosen within each fraction), setting the microscope-video image analysis system at $400 \times$ magnification, and counting all neurons within that tissue volume. The computerized system (JAVA, Jandel) was used to project an image of the striatopallidal area under analysis upon a monitor screen and delineate the borders of each brain fraction in which a fraction was to be sampled. To count the total neurons from the top of the section to the bottom, the counting grid was focused on the surface of the slice and all neurons in focus were counted. The microscope was then adjusted to a deeper plane of focus, so that the images of the counted neurons were blurred and the images of the deeper neurons became clear. Neurons at these deeper levels were added to the count for the total core sample. The entire depth of the section was 'focused through' in this way until every level within the core sample had been counted. The criteria for what constitutes a neuron was identical to the criteria in the methods in the first analysis (circular or ovoid object larger than $10 \mu \mathrm{m}$ in diameter). Neuronal density varied in normal animals from 12 neurons in fractions from the globus pallidus to 154 neurons in fractions from the ventrolateral striatum. However, neuronal density varied by less than $25 \%$ between different rats for the same fraction. This consistency in fraction neuronal number between animals allowed us to set a criterion for the detection of lesions.
Stage 2a. Identifying lesions by the fractionator method

Neuron counts of different control rats varied by less than $25 \%$ for the same fraction. This consistency in the fraction neuronal number between animals allowed us to set a criterion for the detection of lesions. 'Moderate neuron loss' was judged to exist if a fraction had less than $50 \%$ of the normal mean number of neurons for that fraction; this deviation below normal is twice the maximum variation seen between control rats for any one fraction. 'Severe neuron loss' was judged to exist if the fraction had lost at least $80 \%$ of its neurons. Fractions which had the most severe neuron loss were labeled the center of the lesion.

\section{Stage 2b. Lesion border analysis}

Once the center of the lesion had been located, 8 radial arms were drawn using the video image analysis system (JAVA System, Jandel, Corp.) emanating from the center along the major compass points $(0$ degrees, 45 degrees, 90 degrees, 135 degrees etc., see Fig. 4). 'Core sample' counts were taken along each line at 250 $\mu \mathrm{m}$ steps at $400 \times$ magnification (see above) until the neuron density rose above $50 \%$ of the normal level for that fraction. Lesion borders were mapped onto stereotaxic atlas pictures of the representative brain slices $^{28}$ and were traced into the computer using a digitizing tablet and a 3-D reconstruction program (PC3D, Jandel). The digitized slices were converted into a 3-D representation for viewing lesions from a lateral, ventral, and a dorsal 3-D view (see Fig. 6).

\section{Stage 3. Subtraction of non-crucial sites of damage}

In order to determine the crucial site for aversion, defined as the site in which damage was necessary and sufficient for aversion to occur, a composite map of shared damage was made. This was done by adding the mapped lesions (area of depletion above 55\%) of each aversive rat together, and subtracting away any area of damage that was not shared unanimously by the group. The remaining composite lesion identified the common site that was damaged in every rat which displayed enhanced aversion.

\section{Results of fractionator analysis}

Taken as a group, aphagic rats that showed enhanced aversion had unanimous severe damage in a single restricted bilateral area within the VP/SI: the ventromedial corner of the structure, which is adjacent to but outside of the dorsolateral rim of the lateral hypothalamus (see Fig. 5 for an example of a mapped aversion-inducing lesion and Figs. 6 and 7 for common subregion). The stereotaxic center for this lesion was 


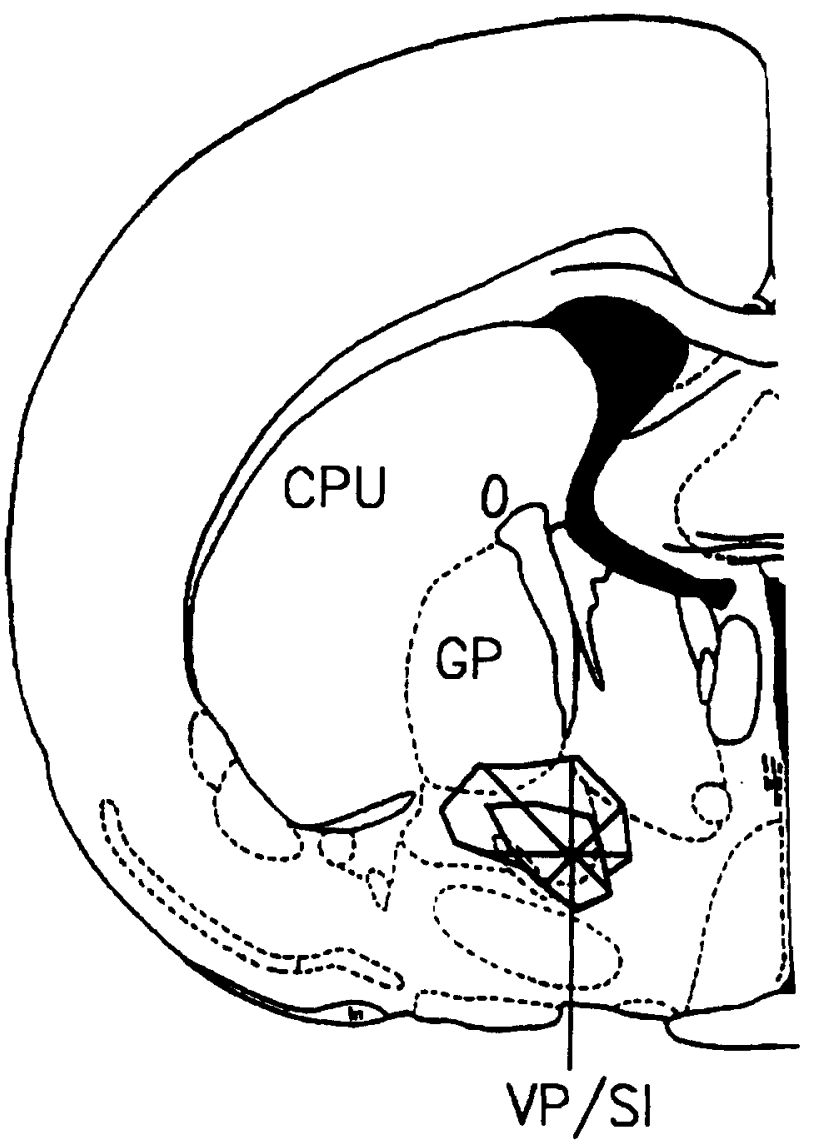

Fig. 5. Mapped lesion for a rat that showed enhanced aversion (right side, inner perimeter denotes border of $>80 \%$ neuron loss; outer perimeter denotes area of $>50 \%$ neuron depletion).

located at $1.3 \mathrm{~mm}$ posterior from bregma (at this AP level each rat had the largest lesion area), $1.9 \pm 0.5 \mathrm{~mm}$ lateral from bregma, and $7.3 \pm 0.25 \mathrm{~mm}$ ventral to the skull. The lateral diameter of the shared lesion site was $1.0 \mathrm{~mm}$, the dorsoventral diameter was $0.5 \mathrm{~mm}$ and the AP diameter was $1.0 \mathrm{~mm}$.

Each rat with enhanced aversion had at least $70 \%$ neuron depletion within this area of the VP/SI. No other structure was damaged bilaterally to this extent in all rats. Only 4 of the rats had bilateral damage in the LH (>50\%). Only 5 of the rats had damage in the GP dorsal to the VP/SI region ( $>60 \%$ neuron loss on average). No other structure in the basal forebrain was damaged in more than 2 of the rats that showed aphagia and enhanced aversion.

\section{DISCUSSION}

There appears to be one crucial anatomical site where lesions produce enhanced aversion, located in the ventromedial corner of the ventral pallidum/ substantia innominata (see Fig. 6). All rats that showed enhanced aversion had significant neuron loss $(\geqq 70 \%$
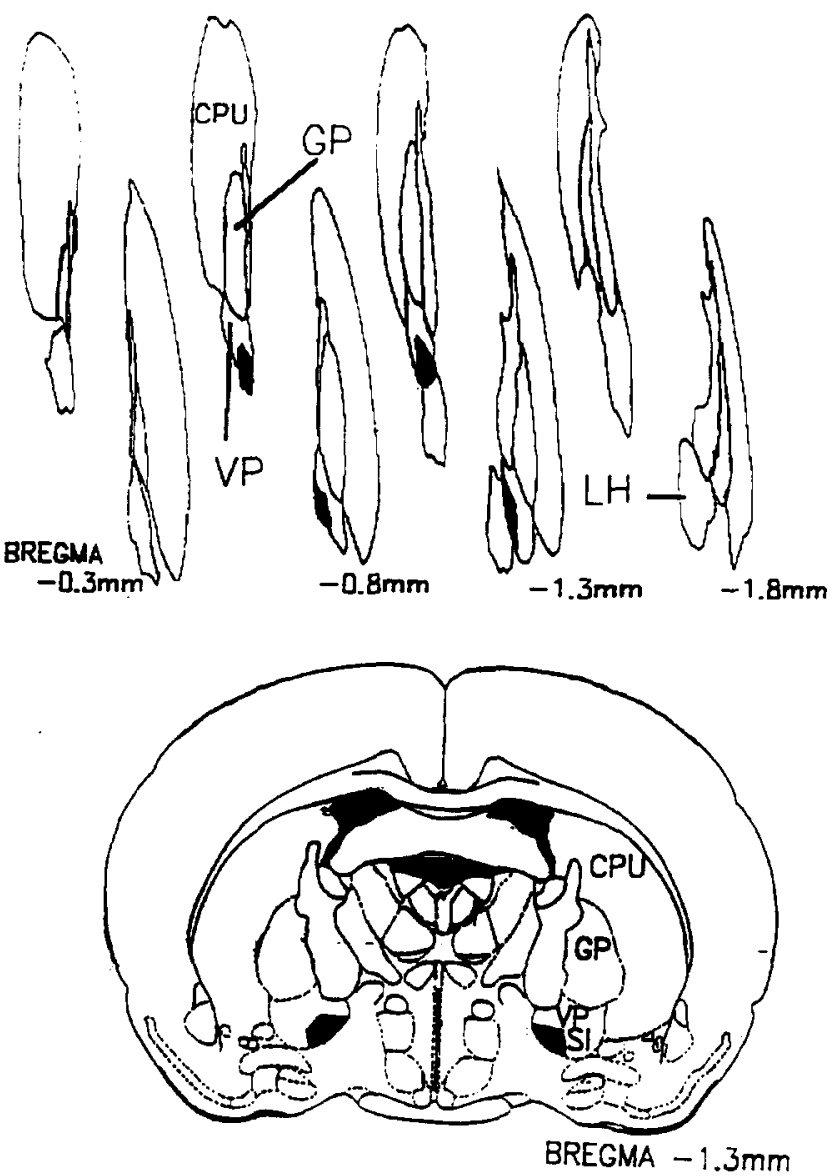

Fig. 6. Three-dimensional (above) and single slice view (below) of the crucial site of damage related to enhanced aversion. Solid region

represents site where all rats had at least $70 \%$ neuron depletion.

for the fractionator analysis) from this site. Out of the eight rats that showed enhanced aversion to sucrose (1 M) only 4 had significant damage in the $\mathrm{LH}$, and even these rats had centers of damage in the VP/SI region.

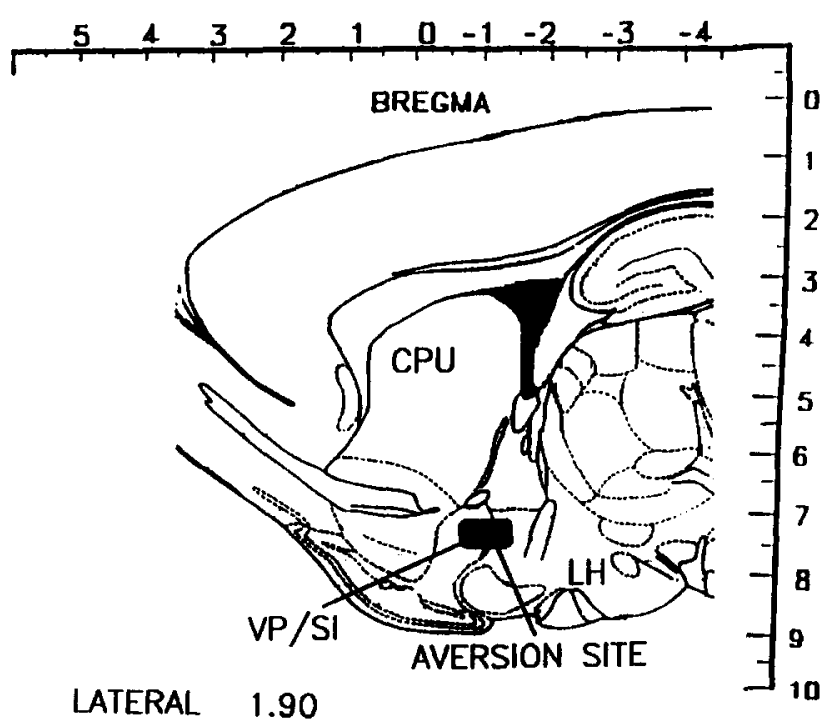

Fig. 7. Sagittal view of the crucial site in which damage produces enhanced aversion (solid region). 
Conversely, none of the rats that had lesions centered within either the GP or the LH showed enhanced aversive responses to sucrose, even though the absolute magnitude of neuronal depletion from both these sites was as great as most aversion-inducing lesions $\geqq 60 \%$ GP, $\geqq 70 \%$ LH; Table I). In LH-centered lesions, neural depletion of the VP/SI aversion site did not exceed $50 \%$ in most rats. Previous reports of 'active aversion' produced by large electrolytic lesions of either the $\mathrm{GP}^{27}$ or the anterior $\mathrm{LH}^{35}$ may actually have depended upon intrusion of the lesion into the ventromedial VP/SI site. The facts that (1) the center of lesions that produced aversion were within the medial VP/SI rather than the LH, (2) several rats that showed aversion had no damage within the $\mathrm{LH}$, and (3) lesions that were centered in the anterior $\mathrm{LH}$ region of Schallert and Whishaw ${ }^{35}$ did not produce aversion, together provide strong evidence that the crucial locus of aversion is outside of the $\mathrm{LH}$.

Why should the ventromedial VP/SI be the site for lesion-induced food aversion? A lesion in the ventral pallidum/substantia innominata could affect neurons belonging to at least four separable neuroanatomical systems: (1) the 'ventral striatopallidal pathway', (2) the 'extended amygdala' system, (3) the cholinergic corticopetal system or (4) gustatory projections to the ventral pallidal/substantia innominata region (see Fig. 8).

\section{Ventral striatopallidal pathway}

The medial portion of the ventral pallidum has been suggested by Heimer and colleagues to differ from the

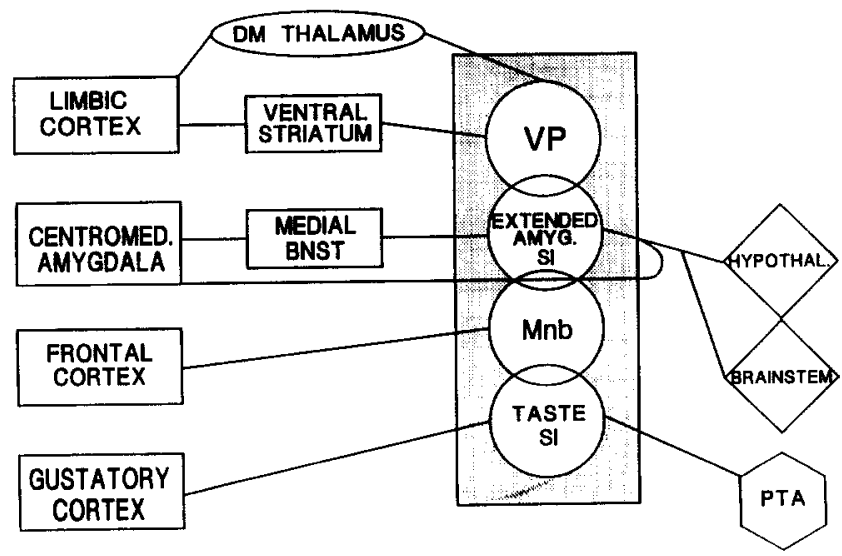

Fig. 8. Some of the basal forebrain subsystems which could be involved in the enhancement of aversion. Area in gray highlights a sample of four of the possible neuronal populations which could be directly affected by the lesion. CENTROMED. AMYGDALA, centromedial amygdala; DM THALAMUS, dorsomedial thalamus; EX TENDED AMYG. SI, extended amygdala system; HYPOTHAL. hypothalamus; MEDIAL BNST, medial bed nucleus of the stria terminalis; Mnb, magnocellular nucleus of the nucleus basalis; PTA, pontine taste area; TASTE SI, taste substantia innominata. rest of the VP in that the "ventromedial VP does not contain typical 'pallidal' cells like those in the dorsolateral part of the VP which are crowded in a typical palisade type fashion by GABAergic and SP or enkephalin-positive boutons; rather the cell bodies and proximal dendrites in the ventromedial part of the VP are contacted by a moderate number of boutons and their axons project primarily to the ventral tegmental area rather than the substantia nigra and subthalamus as is typical for the dorsolateral part of the VP. ${ }^{22}$. This system has been suggested to be involved in motivational functions of the basal ganglia ${ }^{28}$. Lesions to the nucleus accumbens, the main afferent to the VP, however, do not produce aphagia or enhanced aversion ${ }^{12}$. This suggests that it is not disruption of the ventral striatopallidal system per se that leads to aversion. However, it is conceivable that damage to the posterior VP portion of the system identified by this study could lead to a disruption of processing not produced by lesions within anterior regions of the same system.

\section{The medial extended amygdala system}

The boundaries of the medial extended amygdala system $^{1}$ map within the borders of our aversion site. This system consists of projections from the medial amygdaloid nuclei to the medial bed nucleus of the stria terminalis ${ }^{1}$. The medial and rostral portion of the extended amygdala overlaps with the medial accumbens. Caudally, the medial extended amygdala continues in the subcommissural area surrounding the posterior limb of the anterior commissure ${ }^{1}$. Lesions that produce enhanced taste aversion also surround the posterior limb of the anterior commissure in the sublenticular area (see Fig. 6). This means that the extended amygdala system was damaged in every rat that showed enhanced aversion. However, lesions of the central amygdaloid complex, the main afferent to the medial extended amygdala neurons within our site, do not lead to enhanced aversion or aphagia ${ }^{15}$. Therefore disruption of the extended amygdala system per se does not produce enhanced aversion. But just as for the ventral striatopallidal system, it remains possible that damage to the extended amygdala system within our site enhances aversion in a way that damage at an afferent site does not.

\section{The magnocellular corticopetal group}

This system consists of cholinergic neurons within the nucleus basalis and ventral pallidum that receive glutamatergic input from the cortex and possibly the striatum $^{11,14}$. This glutamatergic input could be vital because neurons with these receptors would be directly vulnerable to the neurotoxic effects of the specific 
excitotoxins used here ${ }^{3,33}$. Others have suggested that damage to the cholinergic neurons of the nucleus basalis might produce aphagia and enhanced aversion $^{13,42}$. In the study by Whishaw et al..$^{42}$, ibotenic lesions appeared to have overlapped with the aversion-inducing lesions of the present study. However, chronic injections of atropine, a cholinergic antagonist, used to partially mimic the nucleus basalis lesions, does not produce similar aphagia ${ }^{42}$. This suggests that cholinergic neurons of the nucleus basalis may not be the neurons responsible for the enhancement of aversion observed in the present study. Also, it should be noted that lesions of cortical regions or total decortication (i.e. projection targets of the nucleus basalis) does not lead to enhanced aversion ${ }^{40}$. This observation, as in previous cases, shows that general disruption of the corticopetal system per se does not enhance aversive responses to taste as does damage to the ventromedial VP/SI.

\section{Taste-substantia innominata system}

There is a dense projection of gustatory afferents in the rat from the parabrachial taste area of the dorsal pons to neurons within the ventromedial VP/SI, '... limited to a small zone within and just ventral to the medial third of the internal capsule. ${ }^{29}$. This connection may be reciprocal. Neurons within the medial substantia innominata region have been shown to project to the parabrachial region ${ }^{4,23}$ and to gustatory cortex $^{24}$. Gustatory responses from the neurons within the VP/SI region have been recorded electrophysiologically to tastes such as concentrated sugar solutions ( $20 \%$ glucose or saturated sucrose $)^{10,31}$. Electrophysiological responses to food by neurons in the ventral forebrain are also modulated by sensory-specific satiety ${ }^{32}$. Whether these neurons are driven by direct gustatory projections is unknown, but it is possible that they are a neural substrate for hedonic taste processing. If so, it may be the loss of these 'hedonic' neurons that 'unbalances' taste reactions towards aversion.

Since all of these systems overlap at the site where lesions produce aversion, it is very difficult to choose one system rather than another as responsible for the enhanced aversion observed here. In any case, our results suggest that there is only a single basal forebrain region in which neuronal damage enhances aversive reactions to food. This site is outside the LH proper and is located instead at the ventromedial edge of the ventral pallidum/substantia innominata.

Acknowledgements. This work was supported by a National Institutes of Mental Health pre-doctoral fellowship MH-09838 (H.C.C.) and National Institues of Health Grant NS-23959 (K.C.B.). We thank Mark Barsamian for his help with the rats that received lateral hypothalamic lesions. We thank the many undergraduates who helped complete the neuron counting and histology for both analyses, especially Heather Wells, Rick Ruben, and Rick Roberts.

\section{REFERENCES}

1 Alheid, G.F. and Heimer, L. (1988) New Perspectives in basal forebrain organization of special relevance for neuropsychiatric disorders; the striatopallidal, amygdaloid and corticopetal components of substantia innominata, Neuroscience 27, 1-39.

2 Anand, B.K. and Brobeck, R.J. (1951) Hypothalamic control of food intake in rats and cats, Yale J. Biol. Med. 24, 123-140.

3 Beal, M.F., Ferrante, R.F., Swartz, K.J. and Kowall, N.W. (1991) Chronic quinolinic acid lesions in rats closely resemble Huntington's disease, J. Neurosci. 11, 1649-1659.

4 Berk, M.L. and Finkelstein, J.A. (1982) Efferent connections of the lateral hypothalamus area of the rat: an autoradiographic investigation, Brain Res. Bull. 8, 511-526.

5 Berridge, K.C., Fentress, J.C. and Treit, D. (1988) A triggered hyperkinesia induced in rats by lesions of the corpus striatum, Exp. Neurol. 99, 259-268.

6 Berridge, K.C., Vernier, I.L. and Robinson, T.E., Taste reactivity analysis of 6-hydroxydopamine-induced aphagia: implications for arousal and anhedonia hypotheses of dopamine function, Behav. Neurosci. 103 (1989) 36-45.

7 Berridge, K.C. and Cromwell, H.C. (1990) Motivationalsensorimotor interaction controls aphagia and exaggerated treading after striatopallidal lesions, Behav. Neurosci. 104, 778-795.

8 Berridge, K.C. (1990) Ibotenic acid lesions of the LH blunt affect but do not induce taste aversion, Soc. Neurosci. Abstr. 16, 1245.

9 Brugger, M. (1943) Fresstrieb alls hypothalamisches symptom, Helv. Physiol. Pharmacol. Acta 1, 183-198.

10 Burton, M.J., Mora, F. and Rolls, E.T. (1975) Visual and taste neurones in the lateral hypothalamus and substantia innominata: modulation of responsiveness by hunger, J. Physiol. 252, 50-51.

11 Carnes, K.M., Fuller, T.A. and Price, J. (1990) Sources of presumptive glutamatergic aspartatergic afferents to the magnocellular basal forebrain in the rat, J. Comp. Neurol. 302, 824-852.

12 Cromwell, H.C., Karimipour, D. and Berridge, K.C. (1992) Where is aversion: the ventral pallidum/substantia innominata or lateral hypothalamus?, Soc. Neurosci. Abstr. 18 (1992) 856.

13 Dunnett, S.B., Whishaw, I.Q., Jones, G.H. and Bunch, S.T. (1987) Behavioral, Biochemical, and Histochemical effects of different neurotoxic amino acids injected into nucleus basalis magnocellularis of rats, Neuroscience 20, 653-669.

14 Fuller, T.A., Russhen, F.T. and Price, J.T. (1987) Sources of presumptive glutamatergic/aspartatergic afferents to the rat ventral striatopallidal region, J. Comp. Neurol. 258, 317-338.

15 Galaverna, O.G., Seeley, R.J., Berridge, K.C., Grill, H.J., Schulkin, J. and Epstein, A.N., Lesions of the central nucleus of the amygdala:I. Effects on taste reactivity, taste aversion learning, and sodium appetite, Behav. Brain Res, in press.

16 Gosnell, B.A., Morley, J.E. and Levine, A.S. (1984) Lesions of the globus pallidus and striatum attenuate ketocyclazocine-induced feeding, Physiol. Behav. 33, 349-355.

17 Grill, H.J. and Norgren, R. (1978) The taste reactivity test. I. Mimetic responses to gustatory stimuli in neurologically normal rats, Brain Res. 143, 263-279.

18 Grill, H.J. and Berridge, K.C. (1985) Taste reactivity as a measure of the neural control of palatability, In J.M. Sprague and A.N. Epstein (Eds.), Progress in Psychobiology and Physiological Psychology Vol. 2, Orlando, pp. 1-62.

19 Grossman, S.P., Dacey, D., Halaris, A.E., Collier, T. and Routtenberg, A. (1978) Aphagia and adipsia after preferential destruction of nerve cell bodies in hypothalamus, Science 202, 537-539.

20 Gundersen, H.J., Bagger, P., Bendtsen, T.F., Evans, S.M., Korbo, L., Marcussen, N., Moller, A., Nielsen, K., Nyengaard, J.R. and Pakkenberg, B. (1988) The new stereological tools: dissector, fractionator, nucleator, and point sampled intercepts and their use in pathological research and diagnosis, APMIS 96,857-881.

21 Heimer, L., Zaborsky, L., Zahm, D.S. and Alheid, G.F. (1978) 
The ventral striatopallidothalamic projection. I. The striato-pallidal link originating in the striatal parts of the olfactory tubercle, J. Comp. Neurol. 255, 571-591.

22 Heimer, L., Zahm, D.S., Churchill, L., Kalivas, P.W. and Wohltmann, C. (1991) Specificity in the projection patterns of the acumbel core and shell in the rat, Neuroscience 41, 89-125.

23 Holstege, G., Meiners, L. and Tan, K. (1985) Projections of the bed nucleus of the stria terminalis to the mescencephalon, pons, and medulla oblongata in the cat, Exp. Brain Res. 58, 379-391.

24 Krettek, J.E. and Price, J.L. (1977) Projections from the amygdala complex to the cerebral cortex and the thalamus in the rat and cat, J. Comp. Neurol. 172, 687-722.

25 Lasiter, P.S. and Garcia, J. (1984) A methoxyflurane delivery system for stereotaxic surgery, Brain Res. Bull. 13, 457-460.

26 Levine, M.S. and Schwartzbaum, J.S., Sensorimotor functions for the striatopallidal system and lateral hypothalamus and consummatory behavior in rats, J. Comp. Physiol. Psychol. 85, 615-635.

27 Morgane, P.J. (1961) Alterations in feeding and drinking behavior of rats with lesions of the globus pallidi, Am. J. Physiol. 201, $420-428$.

28 Nauta, W.J.H. (1989) Reciprocal links of the corpus striatum with the cerebral cortex and limbic system: a common substrate for movement and thought?, In W. Meuller (Ed.), Neurology and Psychiatry: a Common Substrate for Movement and Thought? Basal, pp. 43-63.

29 Norgren, R. (1974) Gustatory afferents to the ventral forebrain, Brain Res. 81, 285-295.

30 Paxinos, G. and Watson, C. (1982) The Rat Brain in Stereotaxic Coordinates Academic Press, New York.

31 Rolls, E.T., Burton, M.J. and Mora, F. (1976) Neuronal responses associated with the sight of food, Brain Res. 111, 53-66.

32 Rolls, E.T., Murzi, E., Yaxley, S., Thorpe, S.J. and Simpson, S.J. (1986) Sensory-specific satiety: food specific reduction in responsiveness of ventral forebrain neurons after feeding in the monkey, Brain Res. 368, 79-86.

33 Sanberg, P.R., Calderon, S.F., Giordiano, M., Tew, J.M. and
Norman, A.B. (1989) The quinolinic acid model of Huntington's disease: locomotor abnormalities, Exp. Neurol. 105, 45-53.

34 Sandor, P.. Hajnal, A., Jando, G.. Karadi, Z. and Lenard, L. (1992) Microelectrophoretic application of kainic acid into the globus pallidus: disturbances of feeding behavior, Brain Res. Bull. $28,752-756$

35 Schallert, T. and Whishaw, I.Q. (1978) Two types of aphagia and two types of sensorimotor impairment after lateral hypothalamic lesions: observations in normal weight, dieted, and fattened rats, J. Comp. Physiol. Psychol. 92, 720-741.

36 Stellar, J.R., Brooks, F.H. and Mills, L.E. (1979) Approach and withdrawal analysis of the effects of hypothalamic stimulation and lesions in rats, J. Comp. Physiol. Psychol. 93, 446-466.

37 Stricker, E.M., Swerdloff, A.F. and Zigmond, M.J. (1978) Intrahypothalamic injections of kainic acid produce feeding and drinking deficits in rats, Brain Res. 15 (1978) 470-473.

38 Teitelbaum, P. and Stellar, E. (1954) Recovery from the failure to eat produced by lateral hypothalamic lesions, Science 120 (1954) 894-895.

39 Teitelbaum, P. and Epstein, A.N. (1962) The lateral hypothalamic syndrome: recovery of feeding and drinking after lateral hypothalamic lesions, Psychol. Rev. 69, 74-90.

40 Ungerstedt, U. (1971) Aphagia and adipsia after 6-hydroxydopamine induced degeneration of nigrostriatal dopamine system, Acta Physiol. Scand. 367, 95-122.

41 Whishaw, I.Q., Schallert, T. and Kolb, B. (1981) An analysis of feeding and sensorimotor abilities of rats after decortication, $J$. Comp. Physiol. Psychol. 95, 85-103.

42 Whishaw, I.Q., O'Conner, W.T. and Dunnett, S.B. (1985) Disruption of central cholinergic systems in the rat by basal forebrain lesions or atropine: effects on feeding, sensorimotor behavior, locomotor activity, and spatial navigation, Behav. Brain Res. 17, $103-115$.

43 Winn, P., Tarbuck, A, and Dunnett, S.B. (1984) Ibotenic acid lesions of the lateral hypothalamus: comparison with electrolytic lesion syndrome, Neuroscience 12, 225-240. 